\title{
Article
}

\section{The Electrodynamic Mechanism of Collisionless Multicomponent Plasma Expansion in Vacuum Discharges: From Estimates to Kinetic Theory}

\author{
Vasily Kozhevnikov*(D), Andrey Kozyrev, Aleksandr Kokovin and Natalia Semeniuk (D) \\ Institute of High Current Electronics, The Department of Theoretical Physics, 2/3 Akademichesky Ave., \\ 634055 Tomsk, Russia; kozyrev@to.hcei.tsc.ru (A.K.); alexander.kokovin.desch@gmail.com (A.K.); \\ viliiskoeozero@yandex.ru (N.S.) \\ * Correspondence: Vasily.Y.Kozhevnikov@ieee.org
}

Citation: Kozhevnikov, V.; Kozyrev, A.; Kokovin, A.; Semeniuk, N. The Electrodynamic Mechanism of Collisionless Multicomponent Plasma Expansion in Vacuum Discharges: From Estimates to Kinetic Theory. Energies 2021, 14, 7608. https:// doi.org/10.3390/en14227608

Academic Editor: Pawel Rozga

Received: 21 October 2021

Accepted: 12 November 2021

Published: 14 November 2021

Publisher's Note: MDPI stays neutral with regard to jurisdictional claims in published maps and institutional affiliations.

Copyright: (c) 2021 by the authors. Licensee MDPI, Basel, Switzerland. This article is an open access article distributed under the terms and conditions of the Creative Commons Attribution (CC BY) license (https:/ / creativecommons.org/licenses/by/ $4.0 /)$.

\begin{abstract}
This paper is devoted to the study of collisionless multicomponent plasma expansion in vacuum discharges. Based on the fundamental principles of physical kinetics formulated for vacuum discharge plasma, an answer is given to the following question: What is the main mechanism of cathode plasma transport from cathode to anode, which ensures non-thermal metallic positive ion movement? Theoretical modeling is provided based on the Vlasov-Poisson system of equations for a current flow in a planar vacuum discharge gap. It was shown that the non-thermal plasma expansion is of a purely electrodynamic nature, caused by the formation of a "potential hump" in the interelectrode space and its subsequent movement under certain conditions consistent with plasma electrodynamic transportation. The presented results reveal two cases of the described phenomenon: (1) the dynamics of single-component cathode plasma and (2) multicomponent plasma (consisting of multiple charged ions) expansion.
\end{abstract}

Keywords: vacuum electronics; vacuum arc; vacuum breakdown; computational physical kinetics

\section{Introduction}

Almost a hundred years have passed since Tanberg discovered in his experimental paper [1] that in a vacuum arc discharge, the cathode spot is the source of the plasma jet containing heavy metal ions. It was found that the evaporation rates of the cathode, including heavy ions of the cathode material, were significantly higher than the thermal velocities and amounted to $10^{7} \mathrm{~cm} / \mathrm{s}$. Numerous studies of many cathode materials and various arc lifetimes have shown that the jet contains a mixture of ions of the cathode material with a predominant fraction of one-, two-, and often three-fold charged ions. Beginning with Tanberg's pioneering paper, there were many experiments and reviews (e.g., see [2-4]) citing vast data about ion-directed energy, angular dependence, ion current magnitudes, etc.

Among the key experimental facts established in multiple studies of vacuum discharge, an important role is devoted to the existence of directed ion flows moving toward the anode. Their energies exceed the typical energy value related to the vacuum discharge combustion voltage. In experiments, it was shown that typical ion motion energy is equal to tens and even hundreds of electron volts, while "thermal" energies lie below 3-5 eV [4]. These ions are informally called "ions with anomalously-high energies." Their transfer is accordingly called an "anomalous transport", as they maintain the ability to travel from cathode to anode. It was measured that up to $5-12 \%$ of the total electric charge in arc discharges is carried by "anomalous" ion transportation [4,5].

Proper understanding of "anomalous" ion transport plays a critical role in industrial applications of various arc discharges, such as high-current vacuum switches and ion sources, as well as in technologies for creating surface layers on materials by plasma 
emission sputtering. Currently, three points of view have been formed in the literature (e.g., [6,7]), which supposedly explain this effect:

- Acceleration occurs during phase transitions, causing hydrodynamic and electromagnetic non-uniformities, contact inhomogeneities, or associated instabilities.

- Plasma expansion and acceleration take place due to Joule heating, which leads to a pressure gradient appearance. In fact, such acceleration occurs due to electron-ion and ion-ion friction processes.

- Finally, ions are accelerated by the electric field toward the anode, mainly in a collisionless regime, due to the existence of a non-monotonic distribution of the non-stationary electric potential.

There is also a problem associated with attempts to reveal the dependence of the velocity of the ion plasma component on the ion charge. This problem is associated with both conflicting theoretical points of view and contradictory results from experimental observations. Several authors claim that the ion velocity grows as the ion charge increases. In external fields, the motion of single ions really obeys such a simple pattern due to the influence of the electrostatic force. However, in a vacuum discharge, each separately taken particle moves in a superposition of external fields and the fields created by the other particles. Therefore, the claim that the ion velocity grows as the ion charge increases is not supported experimentally; moreover, the available experiments are contradictory regarding the derived results. Some authors, such as Davies and Miller [3], have shown that the ion velocity linearly increases with the ion charge number. Other studies, such as those conducted by Tsuruta et al. [8] and, more recently, Yushkov et al. [7], claim that ion velocities are independent of ion charge.

In this paper, we advocate the third point of view by showing a fundamental theoretical explanation of the "anomalous" ion transport phenomenon in terms of a fundamental methodology based on computational physical kinetics. We also discuss the more complicated aspects of "anomalous ion transport", such as the multiple charged ions' dynamics and their role in cathode plasma expansion. Without pretending to be a complete problem description, a theoretical explanation of the ion velocity's dependence on its charge is proposed.

\section{Materials and Methods}

As we pay special attention to the fundamental role of physical kinetics in the problem's formulation and its solution, we use an improved model of a vacuum diode that was previously proposed for a vacuum diode with an electron-only emission [9]. According to the chosen methodology, we characterize electrons and multi-charged ions with distribution functions. Let us denote the electron distribution function as $f_{e}$ and denote ion distribution functions as $f_{i}{ }^{+}, f_{i}{ }^{++}, f_{i}{ }^{++} \ldots$ depending on the ion charge of the cathode plasma. Here, we consider two cases of collisionless plasma dynamics: (a) plasma consisting of electrons and single-charged metal ions (such as antimony cathode plasma with $\mathrm{Sb}^{+}$); and (b) more complex plasma consisting of electrons, single- and double-charged ions (zinc cathode plasma $\mathrm{Zn}^{+}$and $\mathrm{Zn}^{++}$). The initial plasma composition was taken from [4].

We consider a one-dimensional vacuum gap of the length $D$, with the cross-sectional area equal to $S$. In this case, electron and ion distribution functions must be parameterized with three independent parameters: the spatial variable $x$, the collinear momentum $p_{x}$ component, and the time variable $t$. Parameterized in this way, the distribution functions obey collisionless kinetic equations (i.e., the Vlasov equations [10]):

$$
\left\{\begin{array}{l}
\frac{\partial f_{e}}{\partial t}+\frac{p_{x}}{m_{e}} \frac{\partial f_{e}}{\partial x}-q E_{x} \frac{\partial f_{e}}{\partial p_{x}}=0 \\
\frac{\partial f_{i}^{+}}{\partial t}+\frac{p_{x}}{m_{i}} \frac{\partial f_{i}^{+}}{\partial x}+q E_{x} \frac{\partial f_{i}^{+}}{\partial p_{x}}=0 \\
\frac{\partial f_{i}^{++}}{\partial t}+\frac{p_{x}}{m_{i}} \frac{\partial f_{i}^{++}}{\partial x}+2 q E_{x} \frac{\partial f_{i}^{++}}{\partial p_{x}}=0 \\
\cdots
\end{array},\right.
$$


where $q$ is the elementary charge, $E_{x}$ is the electric field strength directed collinearly to the $x$-axis, and $m_{e}$ and $m_{i}$ are the rest masses of the electron and metal ion, respectively (here we consider multi-charged ions of the same material, e.g., $\mathrm{Zn}^{+}$and $\mathrm{Zn}^{++}$, so their rest masses are also the same).

It is important to mention that our model does not include collision integrals and is completely collisionless from a general point of view. This suggestion was made to show that collision-induced effects do not play a crucial role in "anomalous" ion transport, anticipating the widespread point of view [6] and advocating the priority of electron-ion and ion-ion frictions as the main mechanisms of the investigating phenomenon.

The system of Equation (1) describes the nonrelativistic ions and electrons' motion in a self-consistent electric field $E_{x}$ (in the no relativistic case and low current densities, the effect of the magnetic field can be neglected). As the applicability condition for the quasi-neutral approximation of cathode plasma cannot be provided at any time points except the initial $t=0$ given by certain initial conditions, the electric field equation has to be solved mutually with (1). To derive it in a self-consistent way, (1) it must be complemented with Poisson's equation for electric potential $\varphi$

$$
\frac{\partial^{2} \varphi}{\partial x^{2}}=-\frac{q}{\varepsilon_{0}}\left(n_{+}+2 n_{++}+3 n_{+++} \cdots-n_{e}\right), E_{x}=-\frac{\partial \varphi}{\partial x},
$$

where $\varepsilon_{0}$ is the vacuum dielectric permittivity, $n_{e}$ is the electron number density, and $n_{+}$, $n_{++}, n_{+++} \ldots$ are the number densities of ions with the corresponding electric charge. As the number of densities are macroscopic plasma characteristics, they can be derived as zero-moments of the corresponding distribution function in the following way:

$$
n_{e}(x, t)=\int_{-\infty}^{\infty} f_{e}\left(x, p_{x}, t\right) d p_{x}, n_{+}(x, t)=\int_{-\infty}^{\infty} f_{i}^{+}\left(x, p_{x}, t\right) d p_{x}, n_{++}(x, t)=\int_{-\infty}^{\infty} f_{i}^{++}\left(x, p_{x}, t\right) d p_{x}, \ldots
$$

To describe all stages of current flow in a vacuum diode, it is necessary to take into account the minimum external electrical circuit for the diode connection. In this model, we will assume that the vacuum diode is connected in series to a voltage source with an electric potential $U(t)$ through the ballast load of $R$. In this case, we can solve Equation (2) directly in quadratures, obtaining the electric field strength and the electric potential in the form of the following expressions:

$$
\begin{gathered}
E_{x}(x, t)=-\frac{[U(t)-j(t) S R]}{D}-\frac{q}{\varepsilon_{0}} \int_{0}^{x}\left[n_{e}\left(x^{\prime}, t\right)-n_{i}\left(x^{\prime}, t\right)\right] d x^{\prime}+\frac{q}{\varepsilon_{0} D} \int_{0}^{D} \int_{0}^{x}\left[n_{e}\left(x^{\prime}, t\right)-n_{i}\left(x^{\prime}, t\right)\right] d x^{\prime} d x, \\
\varphi(x, t)=[U(t)-j(t) S R] \frac{x}{D}+\frac{q x}{\varepsilon_{0} D} \int_{0}^{D} \int_{0}^{x}\left[n_{e}\left(x^{\prime}, t\right)-n_{i}\left(x^{\prime}, t\right)\right] d x^{\prime} d x-\frac{q}{\varepsilon_{0}} \int_{0}^{x} \int_{0}^{x^{\prime}}\left[n_{e}\left(x^{\prime \prime}, t\right)-n_{i}\left(x^{\prime \prime}, t\right)\right] d x^{\prime \prime} d x^{\prime}
\end{gathered}
$$

where $j(t)$ is the diode total current density, which is defined as the sum of the conduction current densities and the electric displacement current density via the following balance equation:

$$
\varepsilon_{0} \frac{\partial E_{x}}{\partial t}+j_{i}-j_{e}=j(t)
$$

where $j_{e}$ and $j_{i}$ are the ion and the electron current densities, respectively, which are calculated as first-moments from the corresponding distribution function using the following equations:

$$
j_{e}(x, t)=q \int_{-\infty}^{\infty} \frac{p_{x}}{m_{e}} f_{e}\left(x, p_{x}, t\right) d p_{x}, j_{i}(x, t)=q \int_{-\infty}^{\infty} \frac{p_{x}}{m_{i}}\left\langle f_{i}^{+}\left(x, p_{x}, t\right)+2 f_{i}^{++}\left(x, p_{x}, t\right)+3 f_{i}^{+++}\left(x, p_{x}, t\right)+\ldots\right\rangle d p_{x}
$$


By integrating the left-hand and right-hand sides of (5) over $x$ from 0 to $D$, one can obtain the following full current density ordinary differential equation:

$$
j(t)-\frac{\varepsilon_{0} R S}{D} \frac{\partial j(t)}{\partial t}=-\frac{\varepsilon_{0}}{D} \frac{\partial U(t)}{\partial t}+\frac{1}{D} \int_{0}^{D}\left[j_{i}-j_{e}\right] d x \equiv \aleph(t),
$$

which has an exact solution with respect to zero initial condition $j(t=0)=0$

$$
j(t)=-\mathrm{e}^{\frac{t}{\tau}} \int_{0}^{t} \aleph\left(t^{\prime}\right) \frac{\mathrm{e}^{-\frac{t^{\prime}}{\tau}}}{\tau} d t^{\prime} .
$$

After substituting (8) into the quadrature formulas (4) for the electric field and the electric potential, we obtain a closed system of Vlasov equations.

To solve the system of Equation (1), proper initial and boundary conditions must be applied. At $t=0$, we suppose the vacuum diode is empty (i.e., $f_{e}=f_{i}^{+}=f_{i}^{++}=f_{i}^{+++}=\ldots=0$ ). To ensure continuous cathode plasma emission following Dirichlet, constant boundary conditions were applied as the following equations:

$$
f_{e}\left(x=0, p_{x}, t\right)=\frac{n_{e 0}}{\sqrt{2 \pi m_{e} T_{e}}} e^{-\frac{p_{x}^{2}}{2 m_{e} T_{e}}}, \quad f_{i}^{+}\left(x=0, p_{x}, t\right)=f_{i}^{++}\left(x=0, p_{x}, t\right)=f_{i}^{+++}\left(x=0, p_{x}, t\right)=\ldots=\frac{n_{i o}^{k}}{\sqrt{2 \pi m_{i} T_{i}}} e^{-\frac{p_{x}^{2}}{2 m_{i} T_{i}}},
$$

where $T_{e}$ and $T_{i}$ denote ion and electron average temperatures, respectively. We use the typical electron temperature of $T_{e} \sim 3 \mathrm{eV}$ and the ion temperature $T_{i} \sim 1 \mathrm{eV}$ for all ion species. The $n_{e 0}$ and $n_{i 0}{ }^{k}$ ( $k$ denotes an ion charge number) are the number densities of the cathode plasma species. They are usually chosen to provide quasi-neutrality of cathode plasma that must be set for fundamental reasons concerning the nature of cathode plasma, namely, to satisfy the $n_{e o} \approx \sum_{k=1}^{N} k n_{i o}^{k}$ condition.

The system of partial differential Equation (1) was solved numerically using the Cheng-Knorr method on a rectangular phase-space grid $\left(x, p_{x}\right)$, with 3000 by 2001 points for the electron's Vlasov equation and 1000 by 201 points for each ion's Vlasov equations, depending on the species number. The computational algorithm used was similar to the one previously used in paper [9]. This computational scheme was implemented in the Mathworks MATLAB environment with embedded high-performance GPU (graphics processing unit) capabilities. The results of the numerical calculations in this section are validated by the additional use of high-resolution time-stepping methods for semiLagrangian solvers [11]. For numerical integration of tabulated functions in expressions (3), (4), (6) and (8), the Simpson method was used.

We consider the vacuum discharge and further breakdown processes to be initiated by applying the voltage $U(t)$ increasing from a zero value to the amplitude value of $U_{0}$. The voltage pulse increases during a short rise time interval equal to $t_{\text {rise. }}$. As far as the simple electric circuit is considered, the ballast load $R$ also needs to be defined.

\section{Results}

In this paper, two sets of computational results in the framework of the theoretical model proposed above are presented. The first type of calculation contains detailed unsteady dynamics of the vacuum breakdown development in a planar discharge gap, where the quasi-neutral cathode plasma consists only of electrons and positive singlecharged metal ions. This composition of cathode plasma is typical of pure lithium, graphite, and antimony cathodes [4]. The vacuum diode metallic plasma composition in [4] is given for a $100 \mathrm{~A}$ arc discharge measured about $100 \mu \mathrm{s}$ after ignition. As was found previously [12], the ion state charge distributions are almost independent of the arc current, so the cathode plasma distributions given in [4] can be widely used in similar computations. Details of calculations with single-charge metallic ions and preliminary conclusions are 
given in Section 3.1. In Section 3.2, the calculations corresponding to the more complex case of current flow in a vacuum diode are considered. Namely, the next case corresponds to quasi-neutral cathode plasma consisting of electrons and a fraction of single- and doublecharged ions. Following extensive experimental data [4], this situation is typical for vacuum discharges with a cathode of magnesium, zinc, germanium, cadmium, indium, selenium, bismuth, lead, and some other materials.

For both simulation sets, we apply the following general set of parameters: $U_{0}=2 \mathrm{kV}$, $t_{\text {rise }}=100 \mathrm{ps}, D=1 \mathrm{~cm}, S=1 \mathrm{~cm}^{2}, T_{e} \sim 3 \mathrm{eV}$ and $T_{i} \sim 1 \mathrm{eV}, R=250$ Ohms, $n_{0}=10^{20} \mathrm{~m}^{-3}$.

\subsection{Plasma Dynamics of Sb Cathode Plasma}

We begin the study of cathode plasma motion and "anomalous" ion transport with the vacuum diode's plasma dynamics consideration for an antimony ( $\mathrm{Sb}$ ) cathode. According to [4], which describes the ion charge state distribution (CSD), the composition of metal plasma consists of single-charged $\mathrm{Sb}_{+}$ions and electrons. In this case, the system of Equation (1) has two Vlasov equations, which were solved numerically for a large time interval of $t_{\text {end }}=1 \mu \mathrm{s}$. At this time interval, the plasma moves to the anode up to the complete discharge gap, bridging and further transition of the process to a stationary current flow, which is determined by the achievement of the total diode current steady-state.

The density plots in Figures 1 and 2 show the detailed dynamics of the plasma components' distribution functions starting from the zero time point $t=0$. The alternation of the frames on density plots is chosen to show the dynamics of the ion distribution function and the electron distribution function consistently. During the first nanosecond $(t \sim 1 \mathrm{~ns})$, the dynamics of the diode plasma do not differ from the current flow pattern for a vacuum diode with a predominant electron cathode emission. This was studied in detail in paper [9]. Electrons arrive at the collector (anode) at the leading edge of the voltage pulse, forming relaxation oscillations of the collector current. The total diode current also oscillates during $t \sim t_{\text {rise }}$ due to the electric displacement current contribution taken into account self-consistently in the Equation (7).

During the initial period of $\sim 1 \mathrm{~ns}$, plasma ions do not move. The thermal energy of the ionic component of the cathode plasma is insufficient to move the ions across the potential barrier. However, as the electrons accelerate by the applied electric field, this creates an excessive negative space charge region (i.e., a so-called virtual cathode) [9]. The presence of this region causes the creation of a corresponding negative electric potential region. In turn, it creates certain conditions for the ions' movement toward the anode; initially, ions stay motionless. Partially, the "virtual cathode" represents a "potential hump" initiating an ion flux from the near-to-cathode surface. The negative potential region never exceeds several tens of volts by absolute value, but this turns out to be sufficient to accelerate ions and fill the gap between the physical cathode and the virtual cathode with dense quasi-neutral cathode plasma during tens of nanoseconds.

To understand the electrodynamic nature of plasma motion, the electric potential time dynamics are provided in Figure 3. The region of the electric potential fall ("potential hump") is abruptly filled with ions, creating a quasi-neutral plasma inside with a number density at the level of cathode emission values $n_{0}$. At the end of this layer, opposite the physical cathode, there is always a fuzzy boundary of the electron emission, whose velocities are much higher than the velocity of movement of the ionic component. Due to the continuity of the electric potential, this leads to the maintenance of the "potential hump" at this boundary. Its presence contributes to the further filling of the discharge gap with quasi-neutral plasma and plasma expansion from cathode to anode. Thus, the plasma expansion has an essentially non-thermal (electrodynamic) physical nature. The characteristic expansion velocities of the plasma bunch are $\sim 10^{6} \mathrm{~cm} / \mathrm{s}$, which, as can be observed from the ion distribution function density plots (Figure 2), correspond to the average ion energies of tens electron volts. 


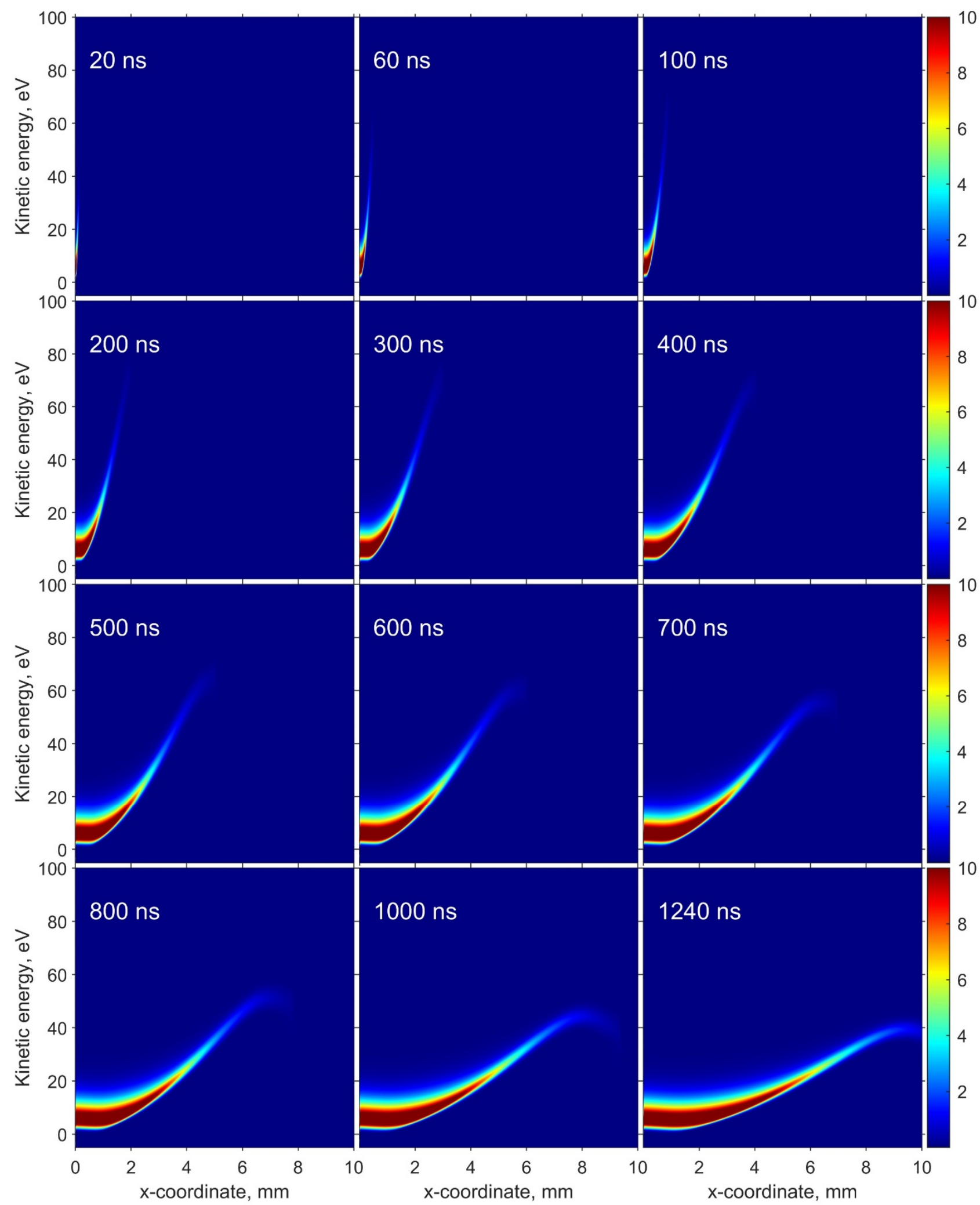

Figure 1. Density plots of the ion $\left(\mathrm{Sb}_{+}\right)$distribution function given at selected time steps for the vacuum breakdown simulation in a diode with antimony cathode. The scale is linear in $10^{39} \mathrm{~s} /\left(\mathrm{kg} \cdot \mathrm{m}^{4}\right)$ units. 


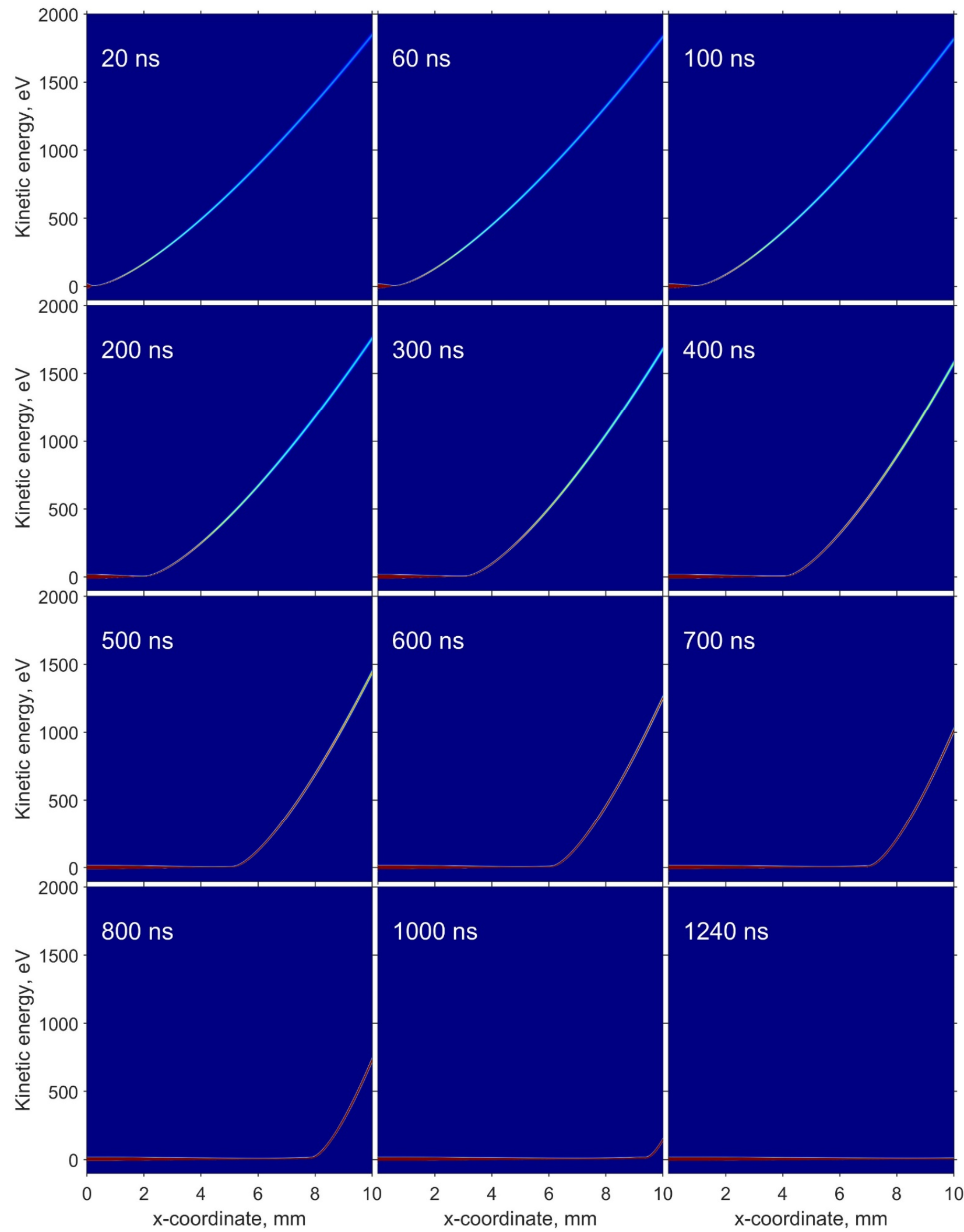

Figure 2. Density plots of the electron distribution function given at selected time steps for the vacuum breakdown simulation in a diode with antimony cathode (arbitrary units). 


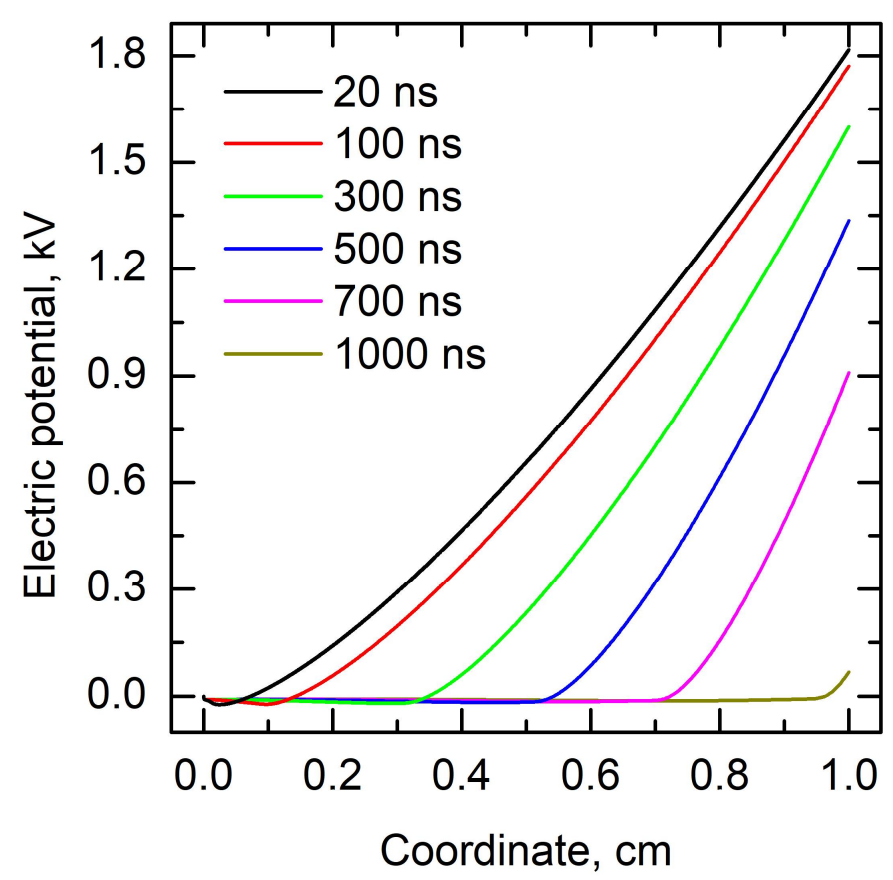

Figure 3. Spatial distribution of the electrostatic potential in vacuum diode with antimony cathode given for selected time steps.

If we consider the time profiles of the electron current density through the collector ((6) at $x=D$ ) and the total current density (8) (in Figure 4), then we can see the difference between them in the stationary current flow section. This difference indicates that up to $12 \%$ of the total electric charge in the discharge we consider is carried by "anomalous" ion transportation. This is in good agreement with the data of experimental papers on the study of vacuum arcs, for example, [5].

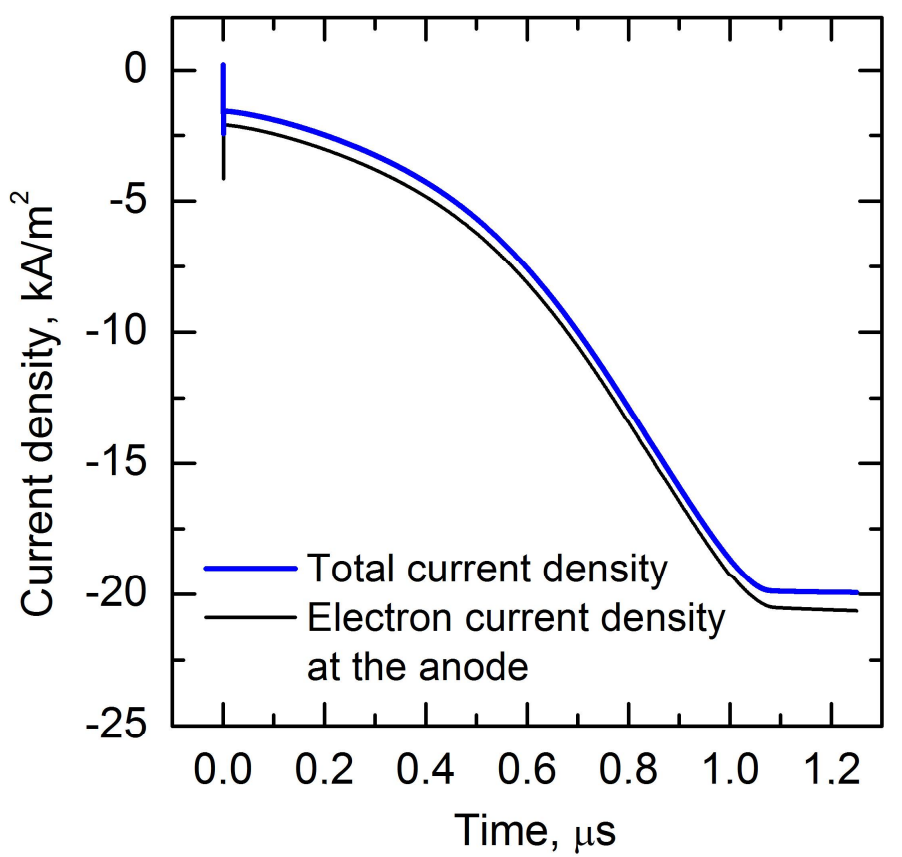

Figure 4. Time profiles of the full diode current density and the electron current density through the collector (anode). 


\subsection{Plasma Dynamics of Zn Multicharged Cathode Plasma}

In the previous subsection, it was convincingly shown that cathode plasma expansion occurs due to the arising of nontrivial conditions that facilitate a continuous electrodynamic acceleration of positive metal ions because the mechanism of "anomalous" ion transport is realized in vacuum diodes. Another important case of collisionless current flow in vacuum diodes is the case of the multicomponent mixture of multiple charged metal ions' expansion. From a general point of view, it is interesting to know the time dynamics of ion fluxes' average velocity depending on the multiplicity of the ion electric charges. Typically, metal cathodes produce multiple charged ions of various charges [4]. For simplicity, we will consider a zinc cathode in this subsection. According to [4], which describes the ion charge state distribution, a vacuum arc in the diode with zinc cathode produces $80 \%$ of $\mathrm{Zn}^{+}$and $20 \%$ of $\mathrm{Zn}^{++}$ions at the steady-state arc glowing regime. This composition of the cathode plasma is specified in the form of stationary boundary conditions (9).

To solve this problem, the system of Equations (1), containing three Vlasov equations, was also solved numerically for a large time interval of $t_{\text {end }}=0.8 \mu \mathrm{s}$. During the defined end time, multicharged cathode plasma moves to the anode, and after complete discharge gap bridging, we obtain a stationary current flow, which is determined by the achievement of the total diode current steady-state value. When simulating, we still neglect all possible mechanisms of ionization and charge exchange of ions, which are of a collisional nature. The solution of kinetic equations is carried out within the framework of a similar numerical calculation scheme.

The dynamics of the electron distribution function are similar to those shown in Figure 2. Figures 5 and 6 show the ion distribution functions for $\mathrm{Zn}^{+}$and $\mathrm{Zn}^{++}$ions, respectively. The general dynamics of the cathode plasma motion from cathode to anode corresponds to the particular case of "anomalous transport" of metallic plasma, which is considered in Section 3.1. At the very beginning, a fast displacement of electrons occurs, an area of "virtual cathode" appears near the physical surface of the cathode, and then, due to the "potential hump" appearance, positive ions fill the area between the physical cathode $(x=0)$ and the virtual cathode. Similar to the previous case (Section 3.1), the potential minimum shifts to the right in a consistent manner as the cathode plasma moves toward the anode. Due to this, "anomalous transport" of ions occurs in plasma with multicharged ions.

At the same time, the given density plots show the difference in the motion dynamics of ions with different charges during the plasma expansion from cathode to anode. The comparison of Figures 5 and 6 indicates that the $\mathrm{Zn}^{++}$component average velocity is almost $20 \%$ higher than the $\mathrm{Zn}^{+}$component velocity. The general movement of the components of the cathode plasma is similar to the previous case, which can be seen in Figure 7, which shows the dynamics of the electric potential in this calculation. This circumstance is due to the fact that the electric force acting on the charged particles is directly proportional to the multiplicity of the electric charge. This, in turn, once again supports the claim that the "anomalous" ions" transportation has a predominantly electrodynamic nature. The observed phenomenology confirms the key results of experimental observations, for example [3], which directly indicate the dependence of the expansion rate of certain plasma components on the ion charge. 


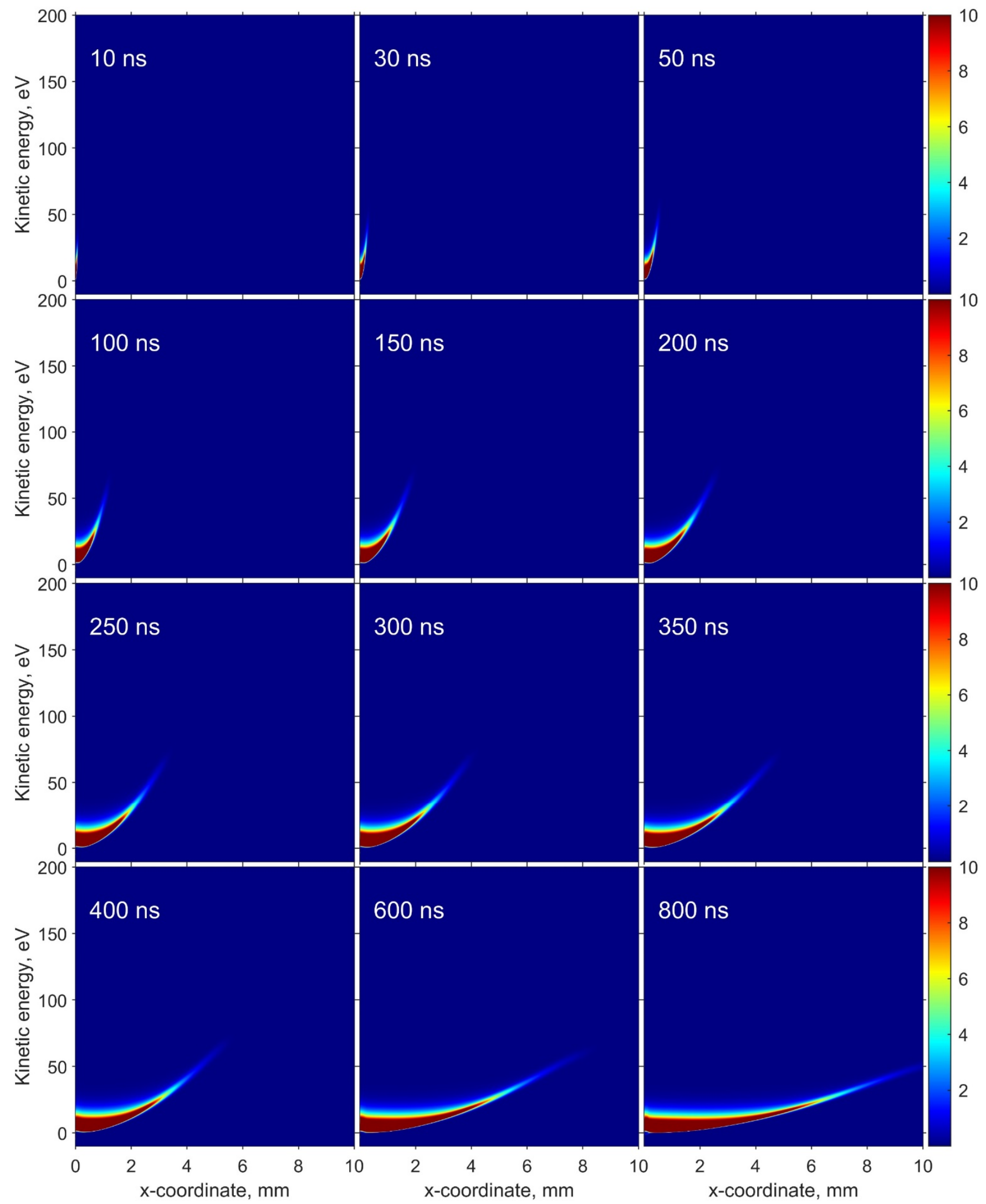

Figure 5. Density plots of the ion $\left(\mathrm{Zn}^{+}\right)$distribution function given at selected time steps for the vacuum breakdown simulation in a diode with zinc cathode. The scale is linear in $10^{39} \mathrm{~s} /\left(\mathrm{kg} \cdot \mathrm{m}^{4}\right)$ units. 


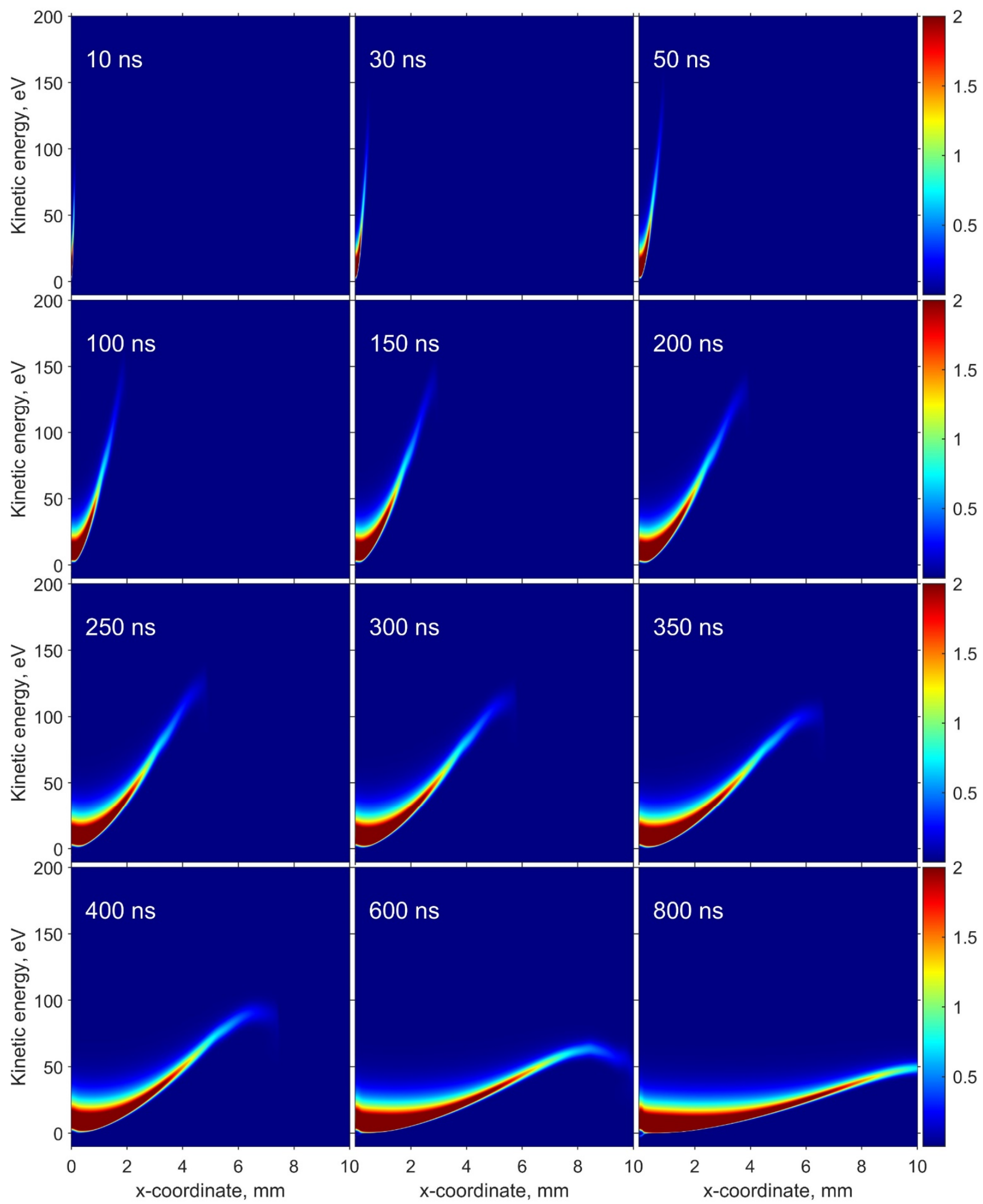

Figure 6. Density plots of the ion $\left(\mathrm{Zn}^{++}\right)$distribution function given at selected time steps for the vacuum breakdown simulation in a diode with zinc cathode. The scale is linear in $10^{39} \mathrm{~s} /\left(\mathrm{kg} \cdot \mathrm{m}^{4}\right)$ units. 


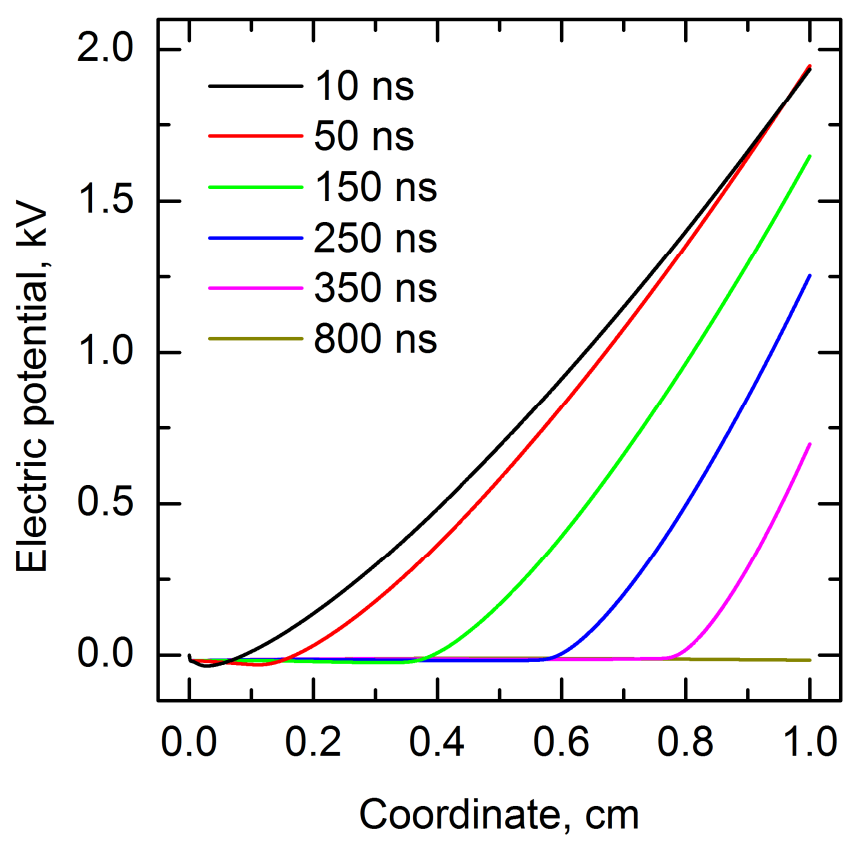

Figure 7. Spatial distribution of the electrostatic potential in vacuum diode with zinc cathode given for selected time steps.

\section{Discussion}

According to the proposed theoretical model based on the fundamental principles of physical kinetics, an explanation of the phenomenon of "anomalous" ion transport of cathode plasma in vacuum diodes was obtained. It was shown that this mechanism has an essentially electrodynamic nature, which was formulated in terms of a collisionless model that self-consistently explains the initial stage of a vacuum breakdown. The considered physical formulation of the problem differs significantly from a previously studied problem pertaining to quasi-neutral plasma bunch expansion in a vacuum [13]. In our problem, a bunch of cathode plasma is placed in a strong external electric field maintained in the interelectrode space; therefore, in contrast to [13], the plasma approximation is inapplicable here. However, the results of our paper indicate that in both cases, the plasma motion (including the "anomalous" ion transport) is predominantly of an electromagnetic nature and is neither collisional (i.e., associated with ion-electron or ion-ion friction) [6] nor associated with a material phase transition [14].

Our model naturally demonstrates at least three facts observed in experiments: (i) ion velocities that nominally exceed the applied voltage (multiplied by the elementary charge) are explained; (ii) the kinetic energy of ions is not proportional to the ion charge $Z$ (in our case, double-charged ions have a kinetic energy of about 1.5 times greater than the single ones); (iii) the fraction of the ion current is about $10 \%$ of the total current.

Author Contributions: Conceptualization, V.K. and A.K. (Andrey Kozyrev); methodology, V.K.; software, V.K. and A.K. (Andrey Kozyrev); validation, N.S., V.K. and A.K. (Andrey Kozyrev); formal analysis, A.K. (Andrey Kozyrev); investigation, V.K., A.K. (Andrey Kozyrev) and A.K. (Aleksandr Kokovin); resources, Aleksandr Kokovin.; data curation, A.K. (Aleksandr Kokovin); writing-original draft preparation, V.K.; writing—review and editing, V.K.; visualization, A.K. (Andrey Kozyrev); supervision, A.K. (Andrey Kozyrev) and V.K.; project administration, A.K. (Aleksandr Kokovin); funding acquisition, A.K. (Aleksandr Kokovin). All authors have read and agreed to the published version of the manuscript.

Funding: The work was carried out within the framework of the state assignment of the Ministry of Science and Higher Education of the Russian Federation on the topics FWRM-2021-0007, FWRM2021-0014. 
Institutional Review Board Statement: Not applicable.

Informed Consent Statement: Not applicable.

Data Availability Statement: No public data sets are used.

Acknowledgments: The authors would like to thank all doctors, nurses and scientists who have found the strength to fight the severe manifestations and consequences of COVID-19. We thank those who spared their time, health and life for sake of defeating the worldwide pandemic for the benefit of all mankind. We also dedicate this paper to all researchers who have died from the effects of COVID-19. The bright memory of them will never be consigned to oblivion and will always be with us.

Conflicts of Interest: The authors declare no conflict of interest.

\section{References}

1. Tanberg, R. On the Cathode of an Arc Drawn in Vacuum. Phys. Rev. 1930, 35, 1080-1089. [CrossRef]

2. Anders, A. Cathodic arcs. In Springer Series on Atomic, Optical, and Plasma Physics; Springer: Berlin/Heidelberg, Germany, 2008.

3. Davis, W.D.; Miller, H.C. Analysis of the Electrode Products Emitted by dc Arcs in a Vacuum Ambient. J. Appl. Phys. 1969, 40, 2212-2221. [CrossRef]

4. Anders, A. Ion charge state distributions of vacuum arc plasmas: The origin of species. Phys. Rev. E 1997, 55, 969-981. [CrossRef]

5. Oks, E.M.; Savkin, K.P.; Yushkov, G.Y.; Nikolaev, A.G.; Anders, A.; Brown, I.G. Measurement of total ion current from vacuum arc plasma sources. Rev. Sci. Instrum. 2006, 77, 03B504. [CrossRef]

6. Krinberg, I.A. Three modes of plasma expansion in a vacuum arc. In Proceedings of the XXIst ISDEIV, Yalta, Ukraine, 27 September-1 October 2004. [CrossRef]

7. Yushkov, G.Y.; Bugaev, A.S.; Krinberg, I.A.; Oks, E.M. On a mechanism of ion acceleration in vacuum arc-discharge plasma. Dokl. Phys. 2001, 46, 307-309. [CrossRef]

8. Tsuruta, K.; Sekiya, K.; Watanabe, G. Velocities of copper and silver ions generated from an impulse vacuum arc. IEEE Trans. Plasma Sci. 1997, 25, 603-608. [CrossRef]

9. Kozhevnikov, V.Y.; Kozyrev, A.V.; Semeniuk, N.S. Modeling of Space Charge Effects in Intense Electron Beams: Kinetic Equation Method Versus PIC Method. IEEE Trans. Plasma Sci. 2017, 45, 2762-2766. [CrossRef]

10. Vlasov, A.A. The vibrational properties of an electron gas. Sov. Phys.-Uspekhi 1968, 10, 721-733. [CrossRef]

11. Yoshida, H. Construction of higher order symplectic integrators. Phys. Lett. A 1990, 150, 262-268. [CrossRef]

12. Paoloni, F.J.; Brown, I.G. Some observations of the effect of magnetic field and arc current on the vacuum arc ion charge state distribution. Rev. Sci. Instrum. 1995, 66, 3855-3858. [CrossRef]

13. Dorozhkina, D.S.; Semenov, V.E. Exact Solution of Vlasov Equations for Quasineutral Expansion of Plasma Bunch into Vacuum. Phys. Rev. Lett. 1998, 81, 2691-2694. [CrossRef]

14. Volkov, N.B.; Nemirovsky, A.Z. The ionic composition of the non-ideal plasma produced by a metallic sphere isothermally expanding into vacuum. J. Phys. D Appl. Phys. 1991, 24, 693-701. [CrossRef] 